\title{
Phasic Activation of Monkey Locus Ceruleus Neurons by Simple Decisions in a Forced-Choice Task
}

\author{
Edwin C. Clayton, ${ }^{1}$ Janusz Rajkowski, ${ }^{1}$ Jonathan D. Cohen, ${ }^{2}$ and Gary Aston-Jones ${ }^{1}$ \\ ${ }^{1}$ Department of Psychiatry, University of Pennsylvania, Philadelphia, Pennsylvania 19104, and ${ }^{2}$ Department of Psychology, Princeton University, Princeton, \\ New Jersey 08544
}

The noradrenergic locus ceruleus (LC) system has been implicated in several behavioral functions, most notably, response to salient sensory events. Here, we provide new evidence indicating a role in the execution of responses associated with simple decisions. We examined impulse activity of monkey LC neurons during performance of a forced-choice discrimination task. The timing of LC activity more closely tracked behavioral responses than stimulus presentation. LC neurons were phasically activated preceding behavioral responses for both correct and incorrect identifications but were not activated by stimuli that failed to elicit lever responses nor by nontask-related lever movements. We hypothesize that the LC responds to the outcome of task-related decision processes, facilitating their influence on overt behavior. This role of the LC in regulating the behavioral outcome of decisional processes contrasts with more traditional views of LC responses as primarily related to sensory processes.

Key words: primate; electrophysiology; norepinephrine; attention; cognition; vigilance

\section{Introduction}

The noradrenergic brain nucleus locus ceruleus (LC) is implicated in a wide variety of CNS processes, including attention (Aston-Jones et al., 1994, 2000; Dalley et al., 2001), arousal (Aston-Jones and Bloom, 1981; Robbins, 1997, 2000), and memory (Arnsten et al., 1988, 1996; Sara and Devauges, 1988; Clayton and Williams, 2000). In general, these have been interpreted in terms of the interaction of the LC with sensory and associative systems innervated by the broad efferent network of LC projections (Foote et al., 1983). However, our laboratory has identified patterns of LC activity indicating that it may have a role in behavioral responses after simple decisions. For example, we found that, during a target-detection task, primate LC cells are phasically activated selectively by infrequent, rewarded target cues but demonstrate little or no response to nontarget distractor stimuli (Aston-Jones et al., 1994). During reversal training (when former nontarget cues became rewarded target cues), the LC shifted its responsiveness to the new target stimulus and became unresponsive to the previously reinforced stimulus (Aston-Jones et al., 1997). These findings indicate that LC activation by targets is not simply a response to the sensory features of the stimuli but instead reflects stimulus meaning and its consequence for behavior. However, it remained unclear whether the phasic LC response was more closely related to stimulus processing or to decision formation and the corresponding behavioral response.

Received Aug. 30, 2003; revised Sept. 15, 2004; accepted Sept. 22, 2004

This work was supported by United States Public Health Service Grants MH55309, MH58480, MH62196, MH12817, and MH12817. We thank Mark Gilzenrat, Philip Holmes, Eric Brown, Jeff Moehlis, and Michiyo Iba for helpful discussions. We also thank Josh Gold for helpful comments on this manuscript.

Correspondence should be addressed to Gary Aston-Jones, Laboratory for Neuromodulation and Behavior, Department of Psychiatry, 705 Stellar-Chance/6100, 422 Curie Boulevard, Philadelphia, PA 19104-6100. E-mail: gaj@mail.med.upenn.edu.

DOI:10.1523/JNEUROSCI.2446-04.2004

Copyright $\odot 2004$ Society for Neuroscience $\quad$ 0270-6474/04/249914-07\$15.00/0
Here we addressed this issue by recording monkey LC activity during a forced-choice discrimination task. This more complex task requires a behavioral response on $80-100 \%$ of trials and produces greater variability in reaction times (RTs) than found previously in our target-detection studies. These characteristics allowed us to conduct a detailed examination of the timing of LC phasic activity in relation to stimulus presentation versus behavioral response. This task also requires a decision about how to respond on each trial, permitting analysis of LC activity with regard to simple decision processes as in several recent studies (Ratcliff, 1978; Ratcliff and Rouder, 1998; Platt and Glimcher, 1999; Schall, 2001; Shadlen and Newsome, 2001; Usher and McClelland, 2001; Gold and Shadlen, 2003; Mazurek et al., 2003). By decision processes, we refer to those processes responsible for mapping the interpretation of a stimulus onto the selection of a corresponding response (i.e., accumulating information regarding the sensory stimulus toward a threshold for eliciting one out of a number of competing responses). We hypothesized that LC phasic activation is more closely tied to the outcome of decisional processes and behavioral responding than to stimulus processing. This hypothesis predicts that LC phasic responses should be more tightly locked to behavioral responses than to stimulus presentation. In contrast, if the primary role of these LC responses is in stimulus processing, as is commonly thought, then the LC phasic response should be more closely related to stimulus onset than to behavioral response. The results support the hypothesis that LC responses are driven by decision processes and may in turn facilitate corresponding behavioral responses.

\section{Materials and Methods}

Forced-choice discrimination task. Two naive adult male rhesus monkeys weighing 13 and $10 \mathrm{~kg}$ were gradually water deprived over a period of $7 \mathrm{~d}$. Subjects were then allowed to consume as much liquid as desired during the testing session but were not supplemented with water after testing. 


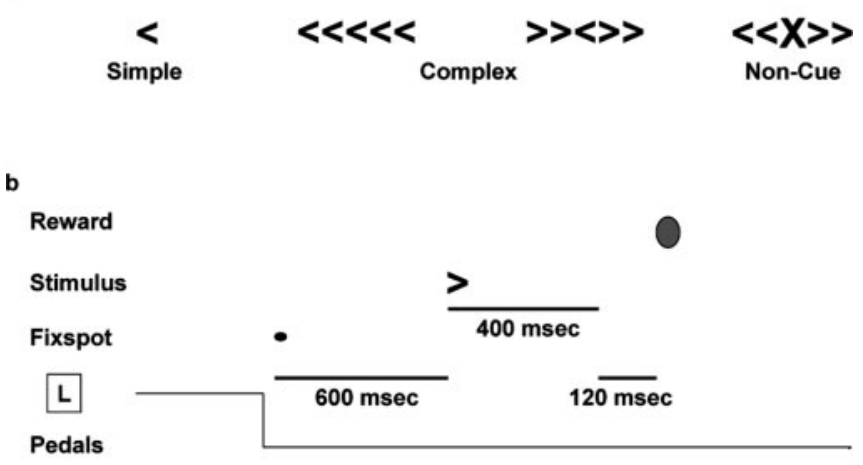

$\mathbf{R}$

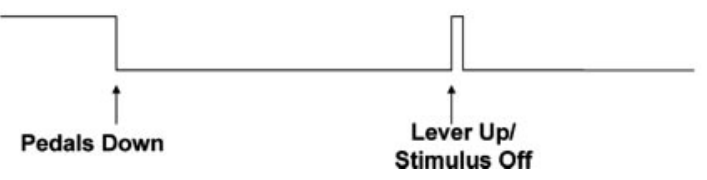

Figure 1. Behavioral stimuli and task progression. $a$, Cues were displayed on a computer screen $(28 \times 21 \mathrm{~cm}) \sim 48 \mathrm{~cm}$ from the monkeys' eyes. Bracket stimuli were identical in size $(21 \times 30 \mathrm{~mm})$ for simple, complex, and noncue stimulus arrays. $b$, Graphical representation of a typical forced-choice trial. After both pedals are depressed ( $R$ and $L$ ), the fixed spot appears and is displayed for $600 \mathrm{msec}$. The animal maintains foveation for the entire period, and then the stimulus is displayed. In the example shown, the subject correctly identifies the right bracket and raises the right lever $400 \mathrm{msec}$ after stimulus onset. The stimulus is then immediately removed from the screen, and, after a brief delay (120 msec), juice is delivered.

On weekends when testing was not conducted, all animals received twice the average amount of fluids consumed during the week. Subjects were weighed daily to ensure that their weight did not drop below $85 \%$ of their pretesting levels.

Subjects were trained to depress two pedals and foveate a centrally located fixation spot on a computer monitor for at least 500-750 msec. After successful fixation, monkeys were presented with either a single visual cue (simple task version) or an array of stimuli consisting of a central cue that was flanked by similar or opposite stimuli (complex version) (Fig. 1). The monkeys were required to make a behavioral response by releasing the lever that corresponded to the directional orientation of the single stimulus or of the central cue in the array. The stimulus was displayed until either the monkey released a lever or $1.2 \mathrm{sec}$ had elapsed, in which case it was considered an omission error. Correct responses were reinforced with a drop of juice reward (Tang) presented 120 msec after lever release; incorrect responses ended the trial, and no reward was available until the following trial. The intertrial interval was typically $\sim 5 \mathrm{sec}$. The response magnitudes $\left(\mathrm{R}_{\text {mags }}\right)$ of the LC phasic responses for the different types of stimuli in the complex task and for simple versus complex tasks were not significantly different, and performance on each task version was also comparable. Therefore, the data for these were combined.

Animals were surgically implanted with a stainless steel guide cannula aimed at the LC, and extracellular recordings from LC neurons were obtained from $25-\mu \mathrm{m}$-diameter, preinsulated stainless steel microwire electrodes as has been described previously (Aston-Jones et al., 1994, 1997). The animals returned to behavioral testing after 1 week for recovery.

After surgery, training in the complex version of the task was slightly altered to include noncue or nonrewarded stimuli on $20 \%$ of the trials in the complex task (75 sessions over 4 months, corresponding to $\sim 4500$ noncue trials, which produced $20 \mathrm{LC}$ recordings). On noncue trials, the central cue was replaced by an " $\mathrm{X}$ " surrounded by the normal bracket flankers (Fig. 1), providing no information about which response lever to use and signaling that reward would not be available on that trial. Nonrewarded trials were identical to normal trials, except that the correct behavioral response was not rewarded. Noncue and nonrewarded trials did not occur during the same day of testing because $>20 \%$ unrewarded (noncue or nonrewarded) trials on a given day decreased overall task performance (i.e., the monkeys stopped responding to task stimuli).

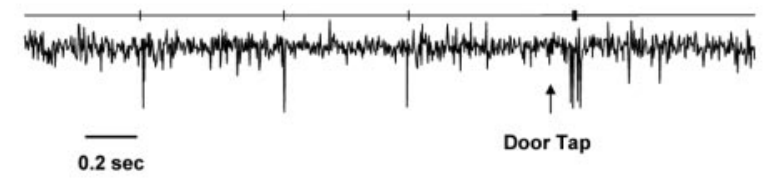

b

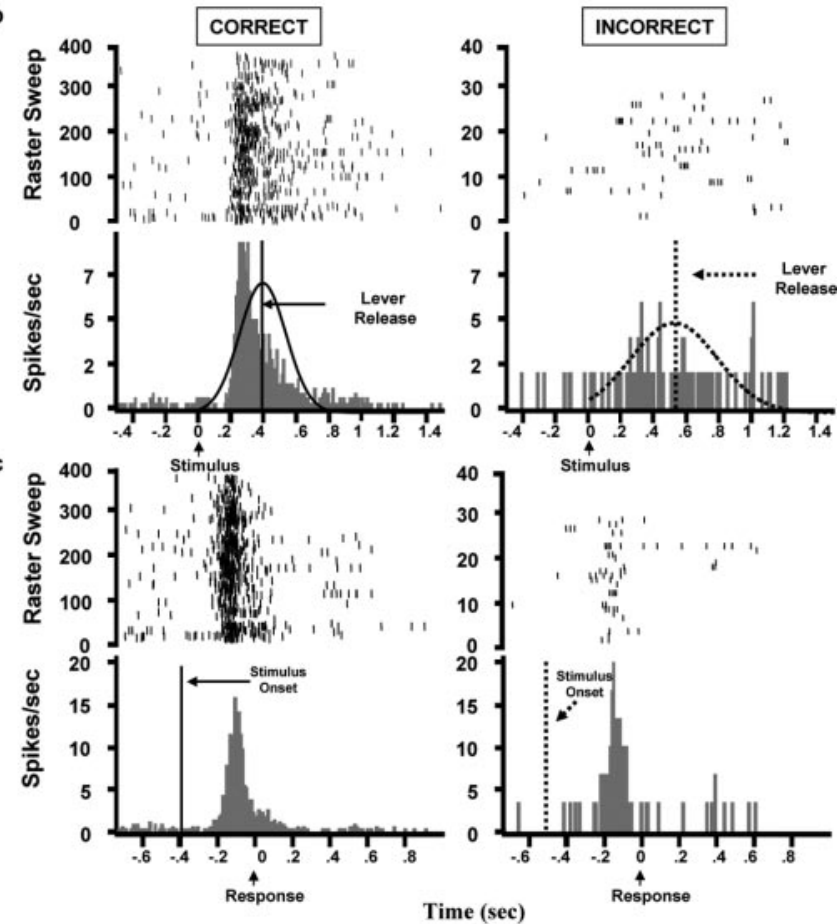

Figure 2. a, Oscilloscope recording of an $\mathrm{LC}$ neuron outside of task performance. An unexpected tap on the experimental chamber produced a phasic $L C$ response, as characteristic of $L C$ neurons in monkeys (Aston-Jones et al., 1994). The line above the trace represents the discriminated LC spikes during this interval. $b, c$, Raster plots and PETHs of activity in a typical single LC neuron depicting stimulus-locked $(b)$ and response-locked $(c)$ activity for trials with correct (367 trials) or incorrect (30 trials) behavioral responses. The same trials were used in these two PETHs. Note that LC activity was more strongly aligned with the behavioral response than with stimulus presentation. This result is most evident when comparing stimulus-locked versus responselocked rasters and PETHs for incorrect trials. Vertical lines indicate the mean RT or stimulus onset, and curves represent the normalized RT distributions for correct and incorrect trials.

Data acquisition and analysis. Perievent time histograms (PETHs) of neural activity were produced offline using Spike2 software (Cambridge Electronics Design, Cambridge, UK). Analyses were focused on neurons with electrophysiological properties characteristic of noradrenergic LC neurons as determined in previous studies (Aston-Jones et al., 1994), including spike form, low-frequency continuous discharge, decreased activity during drowsiness, sensitivity to the $\alpha 2$-noradrenergic agonist clonidine, and a phasic activation-pause response pattern after salient stimuli, such as a tap on the recording booth door (Fig. 2a). In addition, there was no directional bias in either the behavioral or neural data.

To quantitatively examine differences in LC phasic activity between correct and incorrect trials, response magnitude was calculated for individual PETHs as follows: $\mathrm{R}_{\text {mag }}=$ (counts in response interval - baseline activity)/number of trials. Baseline activity was the average discharge rate during the $0.5 \mathrm{sec}$ epoch immediately preceding stimuli for stimuluslocked PETHs, and the response interval examined was 100-400 msec after stimulus. For response-locked histograms, baseline activity was calculated from the first 30 bins $(0.3 \mathrm{sec})$ of the PETH, and the response interval was -300 to $0 \mathrm{msec}$ before the behavioral response. Our previous and current findings indicate that these response intervals contain the bulk of the phasic LC activation. We used this analysis to compare correct versus incorrect trials for individual sessions that contained at least 25 error trials $(n=15)$ because individual PETHs with $<25$ trials 
contained very little LC activity. $\mathrm{R}_{\mathrm{mag}}$ values were calculated from individual-session PETHs and were compared with a $t$ test.

The temporal variability of LC activation in stimulus- and responselocked analyses of correct, incorrect, and noncue trials was also calculated for these individual sessions. For this, the latency of the first LC spike after the beginning of the response interval was determined for each trial, and the mean $\pm \mathrm{SD}$ of these values were determined for each session. The variability of responding for each trial type was calculated as the mean SD of these first-spike latencies. These mean variabilities were then compared by a $t$ test to determine significant differences.

Population PETHs were generated by including activity across multiple sessions. For these population PETHs, counts of corresponding bins in individual-session histograms (not normalized) were summated, and the resulting population PETH was normalized by the total sweep count. Histograms were also smoothed by averaging across three consecutive PETH bins. For comparisons of population histograms, we calculated the onset and peak LC response latencies for each trial type. The onset of excitatory responses in stimulus and response-locked population PETHs was defined as the first bin ( $10 \mathrm{msec}$ bin width) whose value exceeded the mean value of baseline bins by 2 SDs, and that was also the first of five consecutive bins, the average of which was larger than baseline mean +2 SD. The offset of such responses was calculated by finding the first subsequent bin smaller than baseline mean $+2 \mathrm{SD}$ and that was also the first of five consecutive bins whose average was smaller than baseline mean $+2 \mathrm{SD}$.

\section{Results}

Data were compiled over 15 months (testing $\sim 4 \mathrm{~d}$ per week) in which 78 LC recordings were obtained (18 individual neurons and 60 multiunit ensembles) from two rhesus monkeys (57 recordings from monkey B and 21 from monkey C). As found in previous studies (Foote et al., 1983; Aston-Jones et al., 1994, 1997), individual neurons and multiunit ensembles recorded in the LC were very similar for both animals, and therefore their data were pooled. Sessions generally lasted $1.5-2 \mathrm{hr}$, with the subjects receiving 300-800 trials per day and performance ranging from 75 to $98 \%$ accuracy.

\section{Correct versus incorrect trials}

LC neurons exhibited phasic activation shortly after task stimuli, as reported previously for a target-detection task (Aston-Jones et al., 1994, 1997). As seen in Figure 2b, LC activity synchronized with stimulus onset (stimulus-locked PETHs) indicated a stronger LC response on trials with correct behavioral responses than when the animal responded incorrectly. However, examination of LC activity synchronized with the behavioral response (response-locked PETHs) revealed that the magnitudes of phasic LC responses were similar for both correct and incorrect trials (Fig. 2c). Quantitative analysis of individual sessions revealed that LC activation in the response interval of stimulus-locked PETHs for correct trials $\left(R_{\text {mag }}=0.70\right)$ was significantly larger than that for incorrect trials $\left(\mathrm{R}_{\mathrm{mag}}=0.31 ; p=\right.$ $0.04)$. However, there was no significant difference between the magnitudes of LC activation in the response interval for correct versus incorrect trials in response-locked PETHs (1.10 and 0.79, respectively; $p=0.33$ ). Moreover, the magnitude of activation on incorrect trials in response-locked analysis was significantly larger than that for incorrect trials in stimulus-locked PETHs ( $p<$ 0.04).
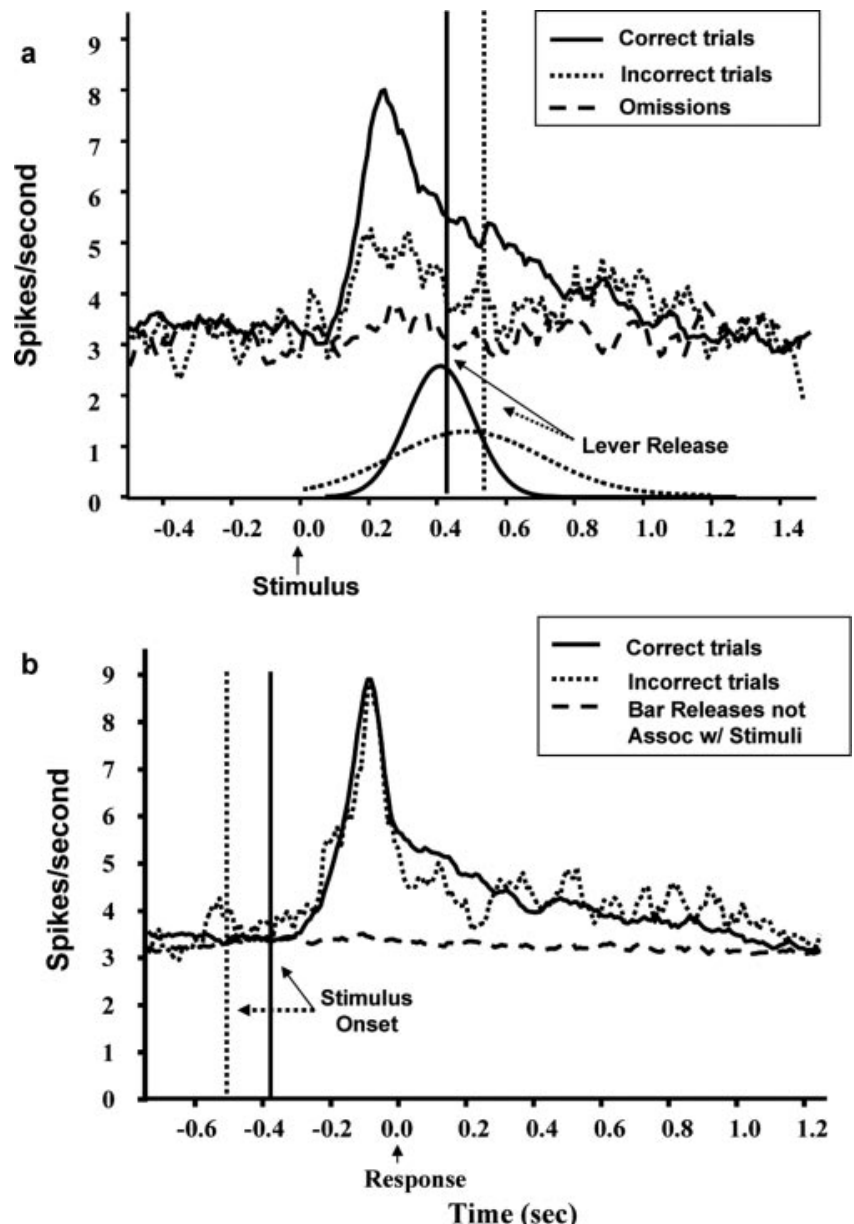

Figure 3. Stimulus- and response-locked population PETHs showing $\mathrm{LC}$ responses to cues for trials yielding correct and incorrect behavioral responses. a, Stimulus-locked population PETHs showing $\mathrm{L}($ response to cues (presented at time 0 ) for trials yielding correct or incorrect behavioral responses. Note that the $\mathrm{LC}$ response peaks sooner and is less prolonged on correct compared with incorrect trials in this analysis (17,533 and 1362 trials, respectively). No fluctuation in LC activity was detected on omission trials (dashed line; 1128 trials). Vertical lines indicate the mean behavioral RTs. Curves represent the normalized RT distributions for correct and incorrect trials. $b$, The difference in the phasic $L C$ response between correct and incorrect trials is no longer evident on response-locked population PETHs. In addition, no LC response occurred before or after lever releases not associated with stimulus presentation (dashed line; 3381 trials). Vertical lines indicate the mean stimulus onset times.

Table 1. Timing (in seconds) of phasic LC and behavioral responses to task cues

\begin{tabular}{|c|c|c|c|c|c|}
\hline \multirow[b]{3}{*}{ PETH } & \multicolumn{4}{|c|}{ Phasic LC response } & \multirow{3}{*}{$\frac{\text { Behavioral }}{\mathrm{RT}(\sec \pm \mathrm{SD})}$} \\
\hline & \multicolumn{2}{|c|}{ Stimulus-locked } & \multicolumn{2}{|l|}{ Response-locked } & \\
\hline & Onset & Peak & Onset & Peak & \\
\hline Individual session correct & $0.22 \pm 0.01$ & $0.35 \pm 0.02$ & $-0.21 \pm 0.02$ & $-0.10 \pm 0.01$ & \\
\hline Population correct & 0.11 & 0.28 & -0.24 & -0.10 & $0.426 \pm 0.127$ \\
\hline Population incorrect & 0.14 & 0.32 & -0.24 & -0.09 & $0.513 \pm 0.207$ \\
\hline Population fast correct & 0.10 & 0.25 & -0.23 & -0.11 & $0.343 \pm 0.034$ \\
\hline Population slow correct & 0.14 & 0.30 & -0.24 & -0.10 & $0.504 \pm 0.140$ \\
\hline Population fast incorrect & 0.15 & 0.26 & -0.23 & -0.10 & $0.376 \pm 0.101$ \\
\hline Population slow incorrect & 0.19 & 1.01 & -0.23 & -0.10 & $0.793 \pm 0.267$ \\
\hline Population nonrewarded & 0.10 & 0.21 & -0.22 & -0.09 & $0.379 \pm 0.117$ \\
\hline Population noncue & 0.16 & 0.26 & -0.24 & -0.09 & $0.549 \pm 0.209$ \\
\hline
\end{tabular}

For stimulus-locked PETHs, values represent $\mathrm{LC}$ response latencies after the visual cue. Latencies for response-locked PETHs reflect the time of $L C$ respons before the lever release. "Individual session correct" values give mean \pm SEM onset and peak latencies for individual LCPETHs (not normalized). This analysis was not feasible for incorrect and noncue data because of the small numbers of these trials in daily sessions. Note the similarities in the response-locked onset and peak values for the individual and population correct PETHs. Onset and peak latencies for the PETHs were rounded to the nearest hundredth of a second because the individual PETHs used to compile each population PETH had a bin width of $0.01 \mathrm{sec}$. 
Population PETHs of correct versus incorrect responses also revealed that the LC response was apparently larger on correct than on incorrect trials in stimulus-locked analyses. However, these LC activations were virtually identical in response-locked PETHs (Fig. 3). These response-locked PETHs revealed that LC responses to forced-choice stimuli consistently preceded behavioral responses by $\sim 0.24 \mathrm{sec}$ (for LC onset and peak activity latencies, as well as RTs for this and all subsequent stimulus and response-locked population analyses, see Table 1).

\section{Omissions and errant lever releases}

Occasionally, the animals failed to respond to stimuli. In marked contrast to other trials, there was no LC response on such omission trials (Fig. $3 a$ ). This lack of phasic LC activity occurred despite the fact that the subjects foveated the stimulus cue for at least $800 \mathrm{msec}$ on $80 \%$ of all omission trials, as indicated by eye position signals. The monkeys' gaze typically did not leave the cue until a behavioral response had been made or until well after a behavioral response would normally have occurred. Analyses of recording sessions divided into $10 \mathrm{~min}$ increments revealed that omissions occurred throughout the testing period and were not associated with time on task.

It is also important to note that lever releases that occurred when the animals were not engaged in task performance (i.e., when no stimuli were present) were not associated with phasic LC activation (Fig. 3b). These movements occurred throughout each daily session and were not associated with time on task or with epochs of poor performance.

\section{Relationship between variabilities of $L C$ activation and behavioral response}

The above results indicated that the diminished LC activation observed in the stimulus-locked incorrect versus correct PETH may be attributable to the variability in the timing of the decision process and the corresponding behavioral response. This hypothesis was supported by analyses revealing that incorrect RTs were not only significantly longer in latency but also considerably more variable than correct RTs (Figs. 2, 3). Additional analyses including all trial types revealed a significant correlation between the RT and the time from stimulus onset to the first LC spike on a trial $(r=0.25 ; p<0.001)$, further indicating that the RT and LC response are closely, temporally linked.

This conclusion was also supported by quantitative trial-bytrial spike latency analyses. These analyses revealed that there was significantly more variability in the time to the first spike in the response interval after stimulus presentation on incorrect than on correct trials (mean SDs $=0.24$ vs $0.15 \mathrm{sec}$, respectively; $p<$ 0.01 ). In contrast, no such difference was found between the variabilities of the latencies of LC responses on incorrect versus correct trials in response-locked analyses (mean SDs $=0.17 \mathrm{vs}$ 0.15 , respectively). In addition, the trial-by-trial variability for LC activation on incorrect trials in response-locked analysis was significantly less than that on incorrect trials in stimulus-locked analysis $(p<0.03)$. These findings support the view that the larger RT variability on incorrect trials accounts for the diminished LC responses on these trials observed in stimulus-locked histograms.

\section{Short- versus long-RT responses}

To further examine the hypothesis that LC activation is linked with the timing of decision processes and behavioral responses, we separated LC data into short and long RTs for both correct and incorrect trials (Fig. 4). Stimulus-locked PETHs revealed that
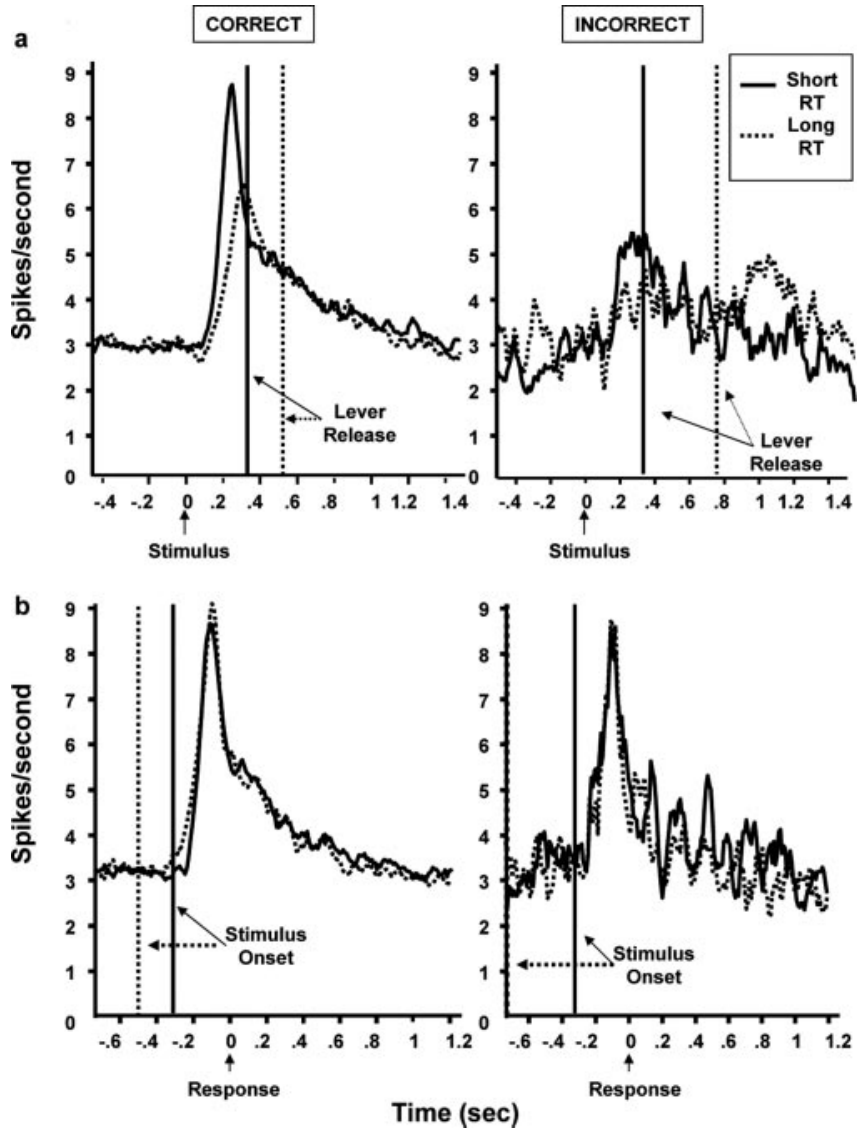

Figure 4. Stimulus-and response-locked population PETHs showing $\mathrm{LC}$ responses to cues on correct or incorrect trials that produced short or long RTs. a, Stimulus-locked population PETHs. Correct trials (left) were divided around the median RT value $(0.388 \mathrm{sec})$ into either short-RT (8098 trials) or long-RT (9435 trials) trials. Onset latencies for $\mathrm{LC}$ responses were similar for both trial types $(0.10-0.14 \mathrm{sec})$, but the peak $\mathrm{LC}$ response occurred sooner on short-RT correct trials $(0.25 \mathrm{sec})$ than for long-RT correct trials $(0.30 \mathrm{sec})$. All incorrect behavioral responses (right) were divided around the median value $(0.479 \mathrm{sec})$ into either short-RT ( 673 trials) or long-RT (689 trials) responses. The size of the $L C$ response is larger on short-RT compared with long-RT incorrect trials $(p<0.01)$. Vertical lines indicate the mean behavioral RT. $b$, Response-locked population PETHs of the same activity as in $a$. Phasic LC activity immediately preceding the lever release was similar for both short- and long-RT correct (left) and incorrect (right) trials. The LC response in this response-locked analysis is quite similar to that observed in the stimulus-locked PETH for the short-RT correct trials, likely attributable to the tight RT distribution for those trials (see Table 1). Conversely, the $L C$ responses in the incorrect response-locked analyses are quite different from those in stimulus-locked PETHs, especially on long-RT incorrect trials, which did not evoke a strong $\mathrm{LC}$ response as seen in the stimulus-locked PETH ( $A$, right). Vertical lines indicate the mean stimulus onset times.

short-RT trials were associated with shorter LC latencies than long-RT trials. For both short- and long-RT trials, the mean behavioral RT consistently followed the peak LC activation in population PETHs. As in the above analyses for correct and incorrect trials, the LC response was more temporally concise, and responses for short- and long-RT trials were more similar in response-locked compared with stimulus-locked PETHs. In addition, regardless of RT, LC response onset occurred $\sim 0.23 \mathrm{sec}$ before the behavioral response.

\section{Noncue and Nonrewarded trials}

For selected sessions, reward was unavailable on $20 \%$ of trials. The unavailability of reward was either signaled (noncue trials) or unsignaled (nonrewarded trials). As seen in Figure 5, the LC phasic activity before the behavioral response on nonrewarded 

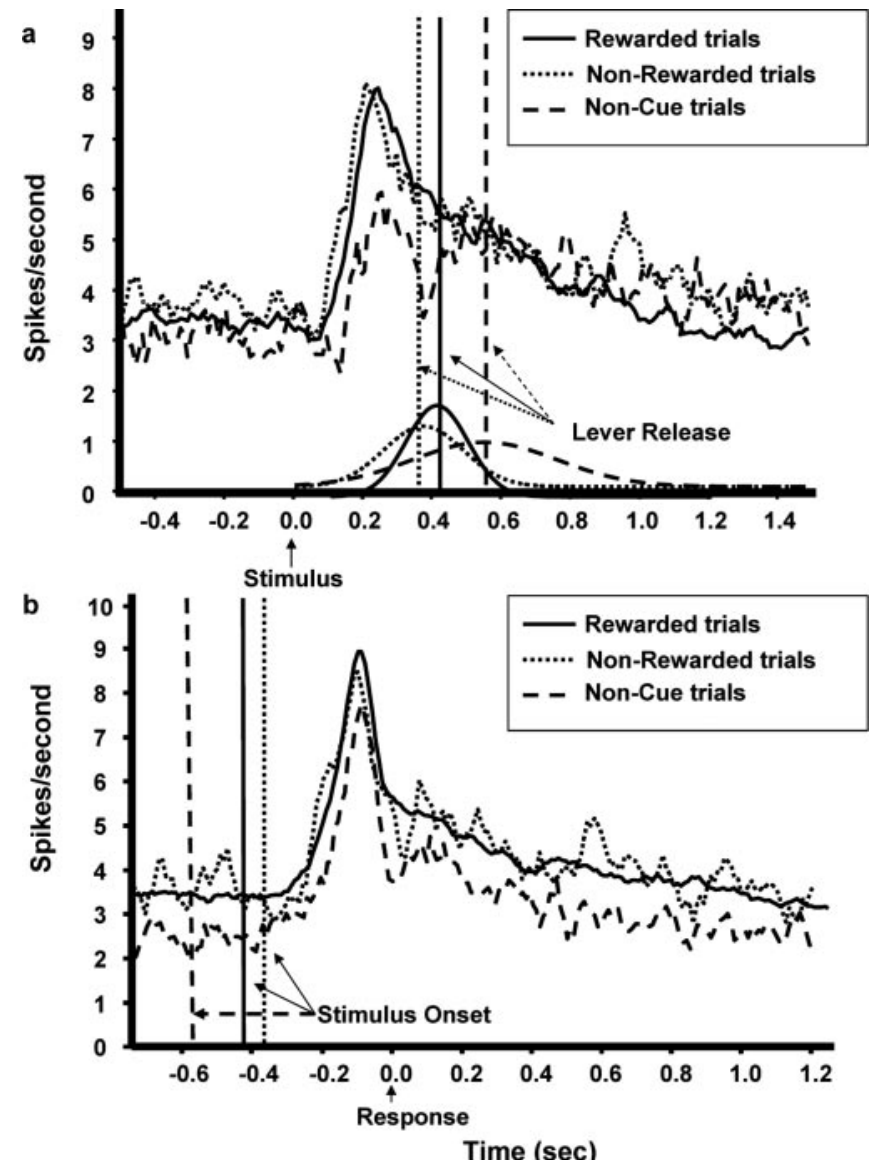

Figure 5. Stimulus-locked and response-locked population PETHs showing LC responses on noncue trials as well as on trials yielding correct behavioral responses to rewardable and nonrewardable stimuli. $a$, Behavioral and $\mathrm{LC}$ responses on rewarded and nonrewarded trials are very similar. The slight difference observed between the two is likely attributable to the limited number of trials included in the nonrewarded analysis (778 nonrewarded trials vs 17,533 rewarded trials). There were too few incorrect nonrewarded trials to analyze these separately. Therefore, this figure represents pooled correct and incorrect nonrewarded trials. Contrary to these findings, on noncue trials (in which the central cue is replaced with an " $X$ "), the RT is significantly longer and the $L C$ response is visibly diminished ( 442 trials). Vertical lines indicate the mean behavioral RTs for days in which noncue or nonrewarded trials were presented. Curves represent the normalized RT distribution for each trial type. $b$, The LC response to noncue stimuli was much more temporally concise when viewed in a response-locked PETH compared with the weaker, prolonged activity observed in the stimulus-locked PETH of $a$. Also, phasic LC activity preceding the behavioral response on noncue and nonrewarded trials was similar to that observed in response-locked PETHs for correct and incorrect trials (Fig. 3b). Vertical lines indicate the mean stimulus onset times.

trials was very similar to that observed on correct (rewarded) trials. In addition, there were no modulations of LC activity after the time of the expected reward when withheld. Although the lack of response to nondelivery of expected reward may be confounded by the immediately preceding LC response, nevertheless it contrasts with brainstem dopaminergic neurons, which show response suppression after the absence of an expected reward (Hollerman and Schultz, 1998).

Stimulus-locked PETHs for noncue trials (in which the lack of reward was signaled) showed a long-latency, variable LC response (Fig. 5a). Correspondingly, the RTs for noncue trials were significantly longer and more variable than for correct rewarded trials occurring on the same testing days $(p<0.05)$. These findings indicate that, as for other types of trials, the variability in the LC response on noncue trials was related to the corresponding
RT variability. Indeed, response-locked PETHs for noncue trials (Fig. 5b) showed stronger phasic LC activation than stimuluslocked PETHs (Fig. 5a) and revealed LC activity similar to that in response-locked PETHs for correct or incorrect trials. In addition, the mean LC response on correct trials was significantly larger than on noncue trials in stimulus-locked trials $(p<0.05)$ but not in response-locked PETHs $(p=0.74)$. Onset and peak LC activation latencies were similar for noncue and correct trials in lever-locked PETHs as well, as seen in Table 1.

\section{Discussion}

This study demonstrates that monkey LC neurons are phasically activated in close temporal relation to the behavioral response in a forced-choice task. Analyses of response-locked versus stimulus-locked PETHs revealed that LC phasic activation was more closely linked to lever releases than to stimulus presentation in correct, incorrect, nonrewarded, noncue, short-RT, and long-RT trials. Response-locked PETHs also indicated that LC response onset precedes behavioral responses by $>0.2 \mathrm{sec}$ in all trial types. In addition, there was no LC response on omission trials or with behavioral responses outside of task performance. Together, these results are consistent with the hypothesis that the LC phasic response is driven by decision-making processes and, in turn, serves to facilitate the behavioral response once a decision has been made.

\section{Relationship to previous findings}

Using a go-no go target-detection task, we found that LC neurons selectively respond to infrequent, attended target stimuli (Aston-Jones et al., 1994). This phasic activation was not dependent on physical stimulus properties and readily switched to a previously nonrewarded nontarget stimulus that was made the target after task reversal (Aston-Jones et al., 1997). The present findings extend those results, showing that the LC is activated after attended stimuli when they are attention-demanding but frequent $(80-100 \%$ of trials) and that these responses do not depend on a go-no go contingency but also occur when behavioral responses are demanded on each trial. Many properties of LC responses are similar during target-detection and forcedchoice performance. (1) Attended stimuli elicit short-latency phasic LC activation that precedes the behavioral response. (2) There is an association between the latencies of LC and behavioral responses, consistent with the hypothesis that LC phasic activity plays a role in the behavioral response. (3) LC responses are not elicited by stimuli that fail to elicit behavioral responses (omission errors) nor by errant lever releases not associated with task performance. This indicates that LC responses are neither sensory nor premotor-motor in nature. These results, and our other recent studies (Rajkowski et al., 2004), indicate that LC responses in a target-detection task may be driven by the outcome of simple decisions.

In addition to these similarities, there were also notable differences in LC responses in these two tasks. In the target-detection task, the phasic activation for target cues was brief in duration $(\sim 0.125 \mathrm{sec})$ and was followed by a period of decreased activity. In the forced-choice task, the response duration was longer, and there was no apparent postactivation inhibition. (However, the absence of apparent postactivation inhibition does not eliminate the possibility that LC neurons are suppressed from further activation immediately after responses.) The reason for these differences is unclear but may reflect the more demanding discrimination compared with the target-detection task. It is also possible that the tonic response inhibition associated with the go-no go 
aspect of the target-detection task was responsible for the abbreviated LC response compared with the forced-choice task.

\section{Response on nonrewarded trials}

Response-locked PETHs reveal strong phasic LC activation preceding the behavioral response to noncue stimuli that signal reward unavailability. This finding indicates that the phasic LC response is not attributable to immediate reward expectation. However, why would the LC respond on trials that signal that no reward is available? One possible explanation is that the LC response facilitates the associated behavioral response, which in turn aborts that trial and decreases the amount of time until the next trial when reward is available. These findings suggest that the LC contributes to the facilitation of the behavioral response even in conditions that do not lead to immediate reinforcement but do improve the likelihood of future reward.

\section{A new theory of LC function}

Previous views held that phasic LC activation functioned to regulate sensory processing. Indeed, in rat, the LC preferentially innervates sensory-related areas in brainstem (Levitt and Moore, 1979), and LC neurons are activated shortly after salient sensory stimuli (Aston-Jones et al., 1984, 1991, 1994; Rasmussen et al., 1986). Other studies showed that LC stimulation or norepinephrine (NE) application increased the responsiveness of neurons in sensory regions to other inputs, supporting the view that LC activation could regulate behavior by altering sensory processing (Devilbiss and Waterhouse, 2000; Waterhouse et al., 2000). However, our current findings demonstrate that the LC response is much more closely linked with the behavioral response than the sensory event.

Together, these findings are consistent with the hypothesis that the LC phasic response is driven by the outcome of the decision process and the commitment to act (Usher et al., 1999), rather than by stimulus presentation. This is equivalent to proposing that LC activation is driven by threshold crossing in accumulator models (including drift-diffusion models) for decision making (Ratcliff, 1978; Ratcliff and Rouder, 1998; Schall, 2001; Usher and McClelland, 2001; Bogacz et al., 2003; Gold and Shadlen, 2003; Mazurek et al., 2003). In this view, variability of the LC response in relation to stimulus onset is attributable to variability in the duration of the decision process (i.e., threshold crossing). Note that this hypothesis does not imply that the LC participates in formulating decisions or adjudicating between alternative responses; rather, we propose that the LC response is driven by the decision made elsewhere. Our ongoing studies reveal similar decision-driven LC activation in a task that uses an oculomotor response, consistent with the hypothesis that the decision process and commitment to act drives these LC responses independent of the specific motor response called for (Iba et al., 2004).

We hypothesize that this decision-driven activation of LC neurons serves to facilitate behavioral responses once the commitment to act has been reached. This hypothesis is supported by the timing of LC phasic activity and impulse conduction. LC responses begin $\sim 230 \mathrm{sec}$ before the lever response, and LC spikes require $\sim 60 \mathrm{msec}$ to reach frontal cortex in monkey (Aston-Jones et al., 1985). Thus, the onset of an LC response would reach the frontal cortex $\sim 170$ msec before the behavioral response, which is approximately the same time as motor cortical circuits are first becoming activated $(\sim 150 \mathrm{msec}$ before a manual behavioral response) (Mountcastle et al., 1972). We propose that the NE liberated in motor circuits would increase synaptic "throughput," as has been reported for NE effects in other areas (Berridge and Waterhouse, 2003), serving to facilitate the behavioral response once the decision has been made. Neural modeling studies support this view, showing that LC responses that are driven by decision processes and that in turn increase the responsiveness of target response elements, substantially increase the rate of reward and facilitate optimal performance in a forcedchoice task (Gilzenrat et al., 2004).

Other possible functions for this LC activation also exist. For example, it could be primarily involved in motor-related reaction time strategies in stimulus-response actions that do not call for high-level cognitive decision processes. Our task uses only simple decision making, and additional studies with complex decision demands are needed to determine the association between LC activation and cognitively complex decision making. It also is possible that reward prediction is important in the LC responses we observed. In fact, in our hypothesis, motivation and reward valence are important contributors to the LC response because they determine which decision processes will drive the LC. In addition, the LC response on trials that signaled reward unavailability does not rule out a role for reward prediction, because it is possible that the monkey misinterpreted the noncue signals and expected reward nonetheless.

It is notable that, although the onset of LC responses precedes lever releases by a sufficient period of time to influence these behavioral responses, later components of the LC activation would produce NE liberation after motor circuits are already activated. This implies that the LC response may serve functions in addition to facilitating the behavioral response. Such functions include learning and memory related to the decision and behavioral response. Substantial evidence indicates that the LC contributes to memory processes (Sara and Devauges, 1988; Arnsten and Contant, 1992; Franowicz et al., 2002). Pharmacological (Klukowski and Harley, 1994) or electrical (Harley, 1991) stimulation of the LC also enhances long-term potentiation, a form of synaptic plasticity associated with memory processing. A role for these LC responses in plasticity is also consistent with evidence that LC activity during waking promotes the expression of plasticity-associated genes in the CNS (Cirelli and Tononi, 2000, 2004). Other possible functions of phasic LC activation include promoting vigilance at the critical time when decision calls for action, consistent with previous views (Aston-Jones et al., 2000; Berridge and Waterhouse, 2003).

The present findings support the hypothesis that the LC plays a role in facilitating the influence of decision processes on behavior. This hypothesis is relevant not only to understanding the role of the LC system in normal cognitive function but also its role in cognitive dysfunctions such as depression (Harro and Oreland, 2001) and attention deficit disorder (Arnsten, 2000). This theory calls for studies of cortical areas responsible for decision-driven LC activity and, conversely, of LC activity and NE release on neural networks related to motor output.

\section{References}

Arnsten AF (2000) Genetics of childhood disorders. XVIII. ADHD, Pt 2, Norepinephrine has a critical modulatory influence on prefrontal cortical function. J Am Acad Child Adolesc Psychiatry 39:1201-1203.

Arnsten AFT, Contant TA (1992) Alpha-2 adrenergic agonists decrease distractibility in aged monkeys performing the delayed response task. Psychopharmacology 108:159-169.

Arnsten AF, Cai JX, Goldman-Rakic PS (1988) The alpha-2 adrenergic agonist guanfacine improves memory in aged monkeys without sedative or hypotensive side effects: evidence for alpha-2 receptor subtypes. J Neurosci 8:4287-4298. 
Arnsten AF, Steere JC, Hunt RD (1996) The contribution of alpha 2-noradrenergic mechanisms of prefrontal cortical cognitive function. Potential significance for attention-deficit hyperactivity disorder. Arch Gen Psychiatry 53:448-455.

Aston-Jones G, Bloom FE (1981) Activity of norepinephrine-containing locus coeruleus neurons in behaving rats anticipates fluctuations in the sleep-waking cycle. J Neurosci 1:876-886.

Aston-Jones G, Foote SL, Bloom FE (1984) Anatomy and physiology of locus coeruleus neurons: functional implications. In: Norepinephrine, Frontiers of clinical neuroscience, Vol 2 (Ziegler M, Lake CR, eds), pp 92-116. Baltimore: Williams and Wilkins.

Aston-Jones G, Foote SL, Segal M (1985) Impulse conduction properties of noradrenergic locus coeruleus axons projecting to monkey cerebrocortex. Neuroscience 15:765-777.

Aston-Jones G, Chiang C, Alexinsky T (1991) Discharge of noradrenergic locus coeruleus neurons in behaving rats and monkeys suggests a role in vigilance. Prog Brain Res 88:501-520.

Aston-Jones G, Rajkowski J, Kubiak P, Alexinsky T (1994) Locus coeruleus neurons in the monkey are selectively activated by attended stimuli in a vigilance task. J Neurosci 14:4467-4480.

Aston-Jones G, Rajkowski J, Kubiak P (1997) Conditioned responses of monkey locus coeruleus neurons anticipate acquisition of discriminative behavior in a vigilance task. Neuroscience 80:697-715.

Aston-Jones G, Rajkowski J, Cohen J (2000) Locus coeruleus and regulation of behavioral flexibility and attention. Prog Brain Res 126:165-182.

Berridge CW, Waterhouse BD (2003) The locus coeruleus-noradrenergic system: modulation of behavioral state and state-dependent cognitive processes. Brain Res Brain Res Rev 42:33-84.

Bogacz R, Moehlis JM, Brown ET, Holmes P, Cohen JD (2003) Neural mechanisms for decision optimization. Soc Neurosci Abstr 29:197.6.

Cirelli C, Tononi G (2000) Differential expression of plasticity-related genes in waking and sleep and their regulation by the noradrenergic system. J Neurosci 20:9187-9194.

Cirelli C, Tononi G (2004) Locus ceruleus control of state-dependent gene expression. J Neurosci 24:5410-5419.

Clayton EC, Williams CL (2000) Glutamatergic influences on the nucleus paragigantocellularis: contribution to performance in avoidance and spatial memory tasks. Behav Neurosci 114:707-712.

Dalley JW, McGaughy J, O'Connell MT, Cardinal RN, Levita L, Robbins TW (2001) Distinct changes in cortical acetylcholine and noradrenaline efflux during contingent and noncontingent performance of a visual attentional task. J Neurosci 21:4908-4914.

Devilbiss DM, Waterhouse BD (2000) Norepinephrine exhibits two distinct profiles of action on sensory cortical neuron responses to excitatory synaptic stimuli. Synapse 37:273-282.

Foote SL, Bloom FE, Aston-Jones G (1983) Nucleus locus ceruleus: new evidence of anatomical and physiological specificity. Physiol Rev 63:844-914.

Franowicz JS, Kessler LE, Borja CM, Kobilka BK, Limbird LE, Arnsten AF (2002) Mutation of the alpha2A-adrenoceptor impairs working memory performance and annuls cognitive enhancement by guanfacine. J Neurosci 22:8771-8777.

Gilzenrat MS, Brown ET, Aston-Jones G, Cohen JD (2004) Locus ceruleus, adaptive gain, and the optimization of decision tasks. Soc Neurosci Abstr 30:899.6.

Gold JI, Shadlen MN (2003) The influence of behavioral context on the representation of a perceptual decision in developing oculomotor commands. J Neurosci Methods 23:632-651.

Harley C (1991) Noradrenergic and locus coeruleus modulation of the perforant path-evoked potential in rat dentate gyrus supports a role for the locus coeruleus in attentional and memorial processes. Prog Brain Res 88:307-321.

Harro J, Oreland L (2001) Depression as a spreading adjustment disorder of monoaminergic neurons: a case for primary implication of the locus coeruleus. Brain Res Rev 38:79-128.

Hollerman JR, Schultz W (1998) Dopamine neurons report an error in the temporal prediction of reward during learning. Nat Neurosci 1:304-309.

Iba M, Clayton EC, Rajkowski J, Aston-Jones G (2004) Decision-related activation of locus coeruleus neurons during performance of an oculomotor delayed-response task. Soc Neurosci Abstr 30:211.2.

Klukowski G, Harley CW (1994) Locus coeruleus activation induces perforant path-evoked population spike potentiation in the dentate gyrus of awake rat. Exp Brain Res 102:165-170.

Levitt P, Moore RY (1979) Origin and organization of brainstem catecholamine innervation in the rat. J Comp Neurol 186:505-528.

Mazurek ME, Roitman JD, Ditterich J, Shadlen MN (2003) A role for neural integrators in perceptual decision making. Cereb Cortex 13:1257-1269.

Mountcastle VB, LaMotte RH, Carli G (1972) Detection thresholds for stimuli in humans and monkeys: comparison with threshold events in mechanoreceptive afferent nerve fibers innervating the monkey hand. J Neurophysiol 35:122-136.

Platt ML, Glimcher PW (1999) Neural correlates of decision variables in parietal cortex. Nature 400:233-238.

Rajkowski J, Majczynski H, Clayton E, Aston-Jones G (2004) Activation of monkey locus coeruleus neurons varies with difficulty and behavioral performance in a target detection task. J Neurophysiol 92:361-371.

Rasmussen K, Morilak DA, Jacobs BL (1986) Single unit activity of locus coeruleus neurons in the freely moving cat. I. During naturalistic behaviors and in response to simple and complex stimuli. Brain Res 371:324-334.

Ratcliff R (1978) A theory of memory retrieval. Psych Rev 85:59-108.

Ratcliff R, Rouder JN (1998) Modeling response times for two-choice decisions: a theory of memory retrieval. Psych Sci 9:347-356.

Robbins TW (1997) Arousal systems and attentional processes. Biol Psychol 45:57-71.

Robbins TW (2000) From arousal to cognition: the integrative position of the prefrontal cortex. Prog Brain Res 126:469-483.

Sara SJ, Devauges V (1988) Priming stimulation of locus coeruleus facilitates memory retrieval in the rat. Brain Res 438:299-303.

Schall JD (2001) Neural basis of deciding, choosing and acting. Nat Rev Neurosci 2:33-42.

Shadlen MN, Newsome WT (2001) Neural basis of a perceptual decision in the parietal cortex (area LIP) of the rhesus monkey. J Neurophysiol 86:1916-1936.

Usher M, McClelland JL (2001) The time course of perceptual choice: the leaky, competing accumulator model. Psych Rev 108:550-592.

Usher M, Cohen JD, Servan-Schreiber D, Rajkowski J, Aston-Jones G (1999) The role of locus coeruleus in the regulation of cognitive performance. Science 283:549-554.

Waterhouse BD, Mouradian R, Sessler FM, Lin RC (2000) Differential modulatory effects of norepinephrine on synaptically driven responses of layer V barrel field cortical neurons. Brain Res 868:39-47. 